\title{
ON WEIGHTED BOCHNER-MARTINELLI RESIDUE CURRENTS
}

\author{
ELIZABETH WULCAN*
}

\begin{abstract}
We study the weighted Bochner-Martinelli residue current $R^{p}(f)$ associated with a sequence $f=\left(f_{1}, \ldots, f_{m}\right)$ of holomorphic germs at $0 \in \mathrm{C}^{n}$, whose common zero set equals the origin, and $p=\left(p_{1}, \ldots, p_{m}\right) \in \mathbf{N}^{m}$. Our main results are a description of $R^{p}(f)$ in terms of the Rees valuations of the ideal generated by $\left(f_{1}^{p_{1}}, \ldots, f_{m}^{p_{m}}\right)$ and an explicit description of $R^{p}(f)$ when $f$ is monomial. For a monomial sequence $f$ we show that $R^{p}(f)$ is independent of $p$ if and only if $f$ is a regular sequence.
\end{abstract}

\section{Introduction}

Let $f=\left(f_{1}, \ldots, f_{m}\right)$ be a sequence of germs of holomorphic functions at $0 \in$ $\mathrm{C}^{n}$, such that $V(f):=\left\{f_{1}=\cdots=f_{m}=0\right\}=\{0\}$. If $f$ is a regular sequence, that is, $m=n$, then there is a canonical residue (current) associated with $f$ - the Grothendieck residue $\operatorname{Res}\left(\frac{\bullet}{f_{1} \ldots f_{m}}\right)$, see [12], and its current avatar, the Coleff-Herrera product $R_{\mathrm{CH}}(f)=\bar{\partial}\left[1 / f_{1}\right] \wedge \cdots \wedge \bar{\partial}\left[1 / f_{m}\right]$, introduced in [9]. In [19] Passare-Tsikh-Yger constructed residue currents based on the BochnerMartinelli kernel as a natural generalization of the Coleff-Herrera product. This idea is further developed in [6], where Berenstein-Yger introduced weighted Bochner-Martinelli residue currents.

Let $p=\left(p_{1}, \ldots, p_{m}\right) \in \mathrm{N}^{m}$ and let $f^{p}$ denote the sequence $\left(f_{1}^{p_{1}}, \ldots, f_{m}^{p_{m}}\right)$; here $\mathrm{N}$ denotes the natural numbers $1,2, \ldots$ For each ordered multi-index $\mathscr{I}=\left\{i_{1}, \ldots, i_{n}\right\} \subseteq\{1, \ldots, m\}$ let

$$
\begin{aligned}
& R_{\mathscr{I}}^{p}(f) \\
= & \left.\bar{\partial}\left|f^{p}\right|^{2 \lambda} \wedge c_{n} \sum_{\ell=1}^{n}(-1)^{\ell-1} \frac{\bar{f}_{i_{\ell}}\left|f_{i_{\ell}}\right|^{2\left(p_{i_{\ell}}-1\right)} \bigwedge_{q \neq \ell}^{\prime} \bar{\partial}\left(\bar{f}_{i_{q}}\left|f_{i_{q}}\right|^{2\left(p_{i_{q}}-1\right)}\right)}{\left|f^{p}\right|^{2 n}}\right|_{\lambda=0},
\end{aligned}
$$

where $c_{n}=(-1)^{n(n-1) / 2}(n-1) !,\left|f^{p}\right|^{2}=\left|f_{1}^{p_{1}}\right|^{2}+\cdots+\left|f_{m}^{p_{m}}\right|^{2}, \bigwedge^{\prime}$ denotes increasing order in $q$ in the wedge product, and $\left.\alpha\right|_{\lambda=0}$ denotes the analytic continuation of the form $\alpha$ to $\lambda=0$. Moreover, let $R^{p}(f)$ denote the vector-valued

\footnotetext{
* The author was partially supported by the Swedish Research Council and NSF grant DMS0901073.

Received 28 September 2009.
} 
current with entries $R_{\mathscr{J}}^{p}(f)$; we will refer to this as the Bochner-Martinelli residue current of weight $p$ associated with $f$. Then $R^{p}(f)$ is a well-defined $(0, n)$-current with support at the origin and $\bar{g} R_{\mathscr{I}}^{p}(f)=0$ if $g$ is a holomorphic function that vanishes at the origin. It follows that the coefficients of the $R_{\mathscr{I}}^{p}(f)$ are just finite sums of holomorphic derivatives at the origin. If $p=(1, \ldots, 1)$, then $R^{p}(f)$ is the Bochner-Martinelli residue current associated with $f$, introduced in [19]; we denote it by $R(f)$ and its entries by $R_{\mathscr{I}}(f)$. Note that, in fact,

$$
R_{\mathscr{I}}^{p}(f)=f_{i_{1}}^{p_{i_{1}}-1} \ldots f_{i_{n}}^{p_{i_{n}}-1} R_{\mathscr{I}}\left(f^{p}\right)
$$

Indeed, the sequence $f^{p}$ in the factor $\bar{\partial}\left|f^{p}\right|^{2 \lambda}$ in (1.1) can be replaced by any sequence of functions that vanish at the origin.

Let $\mathcal{O}_{0}^{n}$ be the local ring of germs of holomorphic functions at $0 \in \mathrm{C}^{n}$. Given a germ of a current $\mu$ at $0 \in \mathrm{C}^{n}$, let ann $\mu$ denote the (holomorphic) annihilator ideal of $\mu$, that is, ann $\mu=\left\{h \in \mathcal{O}_{0}^{n}, h \mu=0\right\}$. Our first result concerns ann $R^{p}(f)$. Let $a(f)$ denote the ideal generated by the $f_{i}$ in $\mathscr{O}_{0}^{n}$. Recall that $h \in \mathcal{O}_{0}^{n}$ is in the integral closure of $a(f)$, denoted by $\overline{a(f)}$, if $|h| \leq C|f|$, for some constant $C$. Moreover, recall that $a(f)$ is a complete intersection ideal if it can be generated by $n=\operatorname{codim} V(f)$ functions. Note that this condition is slightly weaker than that $f$ is a regular sequence. Also, recall that, given ideals $\mathfrak{a}, \mathfrak{b} \subseteq \mathcal{O}_{0}^{n}$, the colon ideal $\mathfrak{a}: \mathfrak{b}$ is the ideal $\mathfrak{a}: \mathfrak{b}=\left\{h \in \mathcal{O}_{0}^{n}: h \mathfrak{b} \subseteq \mathfrak{a}\right\}$.

We also provide a characterization of the non-vanishing entries of $R^{p}(f)$. Let $\pi: X \rightarrow\left(C^{n}, 0\right)$ be a log-resolution of $a(f)$, see [16, Def. 9.1.12]. Following [15] we say that a multi-index $\mathscr{I}=\left\{i_{1}, \ldots, i_{n}\right\}$ is essential with respect to $f$ if there is an exceptional prime $E \subseteq \pi^{-1}(0)$ of $X$ such that the mapping $\left[f_{i_{1}} \circ \pi: \ldots: f_{i_{n}} \circ \pi\right]: E \rightarrow \mathrm{CP}^{n-1}$ is surjective and moreover $\operatorname{ord}_{E}\left(f_{i_{k}}\right) \leq \operatorname{ord}_{E}\left(f_{\ell}\right)$ for $1 \leq k \leq n, 1 \leq \ell \leq m$, see Section 2 and also [15, Section 3] for details. The valuations $\operatorname{ord}_{E}$ that satisfy this are precisely the Rees valuations of $a(f)$. We say that $\mathscr{I}$ is $p$-essential if it is essential with respect to $f^{p}$. For $h \in \mathcal{O}_{0}^{n}$, let $(h)$ denote the ideal generated by $h$.

THEOREM A. Suppose that $f$ is a sequence of germs of holomorphic functions at $0 \in C^{n}$, such that $V(f)=\{0\}$. Let $R^{p}(f)$ be the corresponding Bochner-Martinelli residue current of weight $p$. Then the entry $R_{\mathscr{I}}^{p}(f) \not \equiv 0$ if and only if $\mathscr{I}$ is p-essential. Moreover

$$
\bigcap_{\mathscr{I} p \text {-essential }} \overline{a\left(f^{p}\right)^{n}}:\left(f_{i_{1}}^{p_{i_{1}}-1} \ldots f_{i_{n}}^{p_{i_{n}}-1}\right) \subseteq \operatorname{ann} R^{p}(f) \subseteq \mathfrak{a}(f) .
$$

The left inclusion in (1.3) is strict whenever $n \geq 2$. If the right inclusion is an equality, then $\mathrm{a}(f)$ is a complete intersection ideal. 
The new results in Theorem A are the characterization of the non-vanishing entries and the last two statements. Berenstein-Yger [6] showed that $\overline{\mathfrak{a}\left(f^{p}\right)^{n}}:\left(f_{i_{1}}^{p_{i_{1}}} \ldots f_{i_{n}}^{p_{i_{n}}}\right) \subseteq$ ann $R_{\mathscr{I}}^{p}(f)$, and it is easy to see from Andersson's construction of residue currents in [1] that the right inclusion in (1.3) holds. In fact, Berenstein-Yger defined currents $R_{\mathscr{I}}^{p}(f)$ also when $\operatorname{dim} V(f)>0$. The inclusions in (1.3) hold true also in this case, and one can replace the leftmost ideal by $\bigcap_{\mathscr{I}=\left\{i_{1}, \ldots, i_{\mu}\right\}} \overline{a\left(f^{p}\right)^{\mu}}:\left(f_{i_{1}}^{p_{i_{1}}-1} \ldots f_{i_{\mu}}^{p_{i_{\mu}}-1}\right)$, where $\mu=\min (m, n)$.

Also, for $R(f)=R^{(1, \ldots, 1)}(f)$ Theorem A was proved in parts in [19], [1], and [15]. If $f$ is a regular sequence, then the only entry $R_{\{1, \ldots, m\}}(f)$ of $R(f)$ coincides with the Coleff-Herrera product $R_{\mathrm{CH}}(f)$, whose annihilator ideal is precisely $a(f)$, see [10], [18]. This should be compared to [12, Chapter 5.1] where $\operatorname{Res}\left(\frac{\bullet}{f_{1} \ldots f_{m}}\right)$ is defined using the Bochner-Martinelli kernel. The idea of regarding (complete intersection) ideals of holomorphic functions as the annihilator ideals of certain residue currents is central for many applications, see, for example, [7]. For $p=(1, \ldots, 1)$, the inclusions (1.3) read $\overline{\mathfrak{a}(f)^{n}} \subseteq$ ann $R(f) \subseteq \mathfrak{a}(f)$, which gives a direct proof of the Briançon-Skoda Theorem [8]: $\overline{\mathfrak{a}(f)^{n}} \subseteq \mathfrak{a}(f)$. For other applications of Bochner-Martinelli residue currents, see for example [3], [4], and [22].

Weighted Bochner-Martinelli residue currents were introduced in [6] as a tool to construct Green currents but also as a natural extension of BochnerMartinelli residue currents in the spirit of Lipman [17]; the currents have been further studied in [5] and [24]. In the monograph [17] not only the residue $\operatorname{Res}\left(\frac{\bullet}{f_{1} \ldots f_{m}}\right)$ associated with a sequence $f$ plays a role but also residues of the form $\operatorname{Res}\left(\frac{f_{1}^{p_{1}-1} \ldots f_{m}^{p_{m}-1} \bullet}{f_{1}^{p_{1}} \ldots f_{m}^{p_{m}}}\right)$. The currents $R^{p}(f)$ can thus be seen as analogues of these residues. If $f$ is a regular sequence, then $\operatorname{Res}\left(\frac{f_{1}^{p_{1}-1} \ldots f_{m}^{p_{m-1}} \bullet}{f_{1}^{p_{1} \ldots f_{m}^{p_{m}}}}\right)=\operatorname{Res}\left(\frac{\bullet}{f_{1} \ldots f_{m}}\right)$, which in current language reads

$$
f_{1}^{p_{1}-1} \ldots f_{m}^{p_{m}-1} R_{\mathrm{CH}}\left(f^{p}\right)=R_{\mathrm{CH}}(f) .
$$

It follows that $R^{p}(f)$ is independent of $p$ if $f$ is a regular sequence. In general, however, $R^{p}(f)$ depends in an essential way on $p$; the set of non-vanishing entries as well as ann $R^{p}(f)$ depend on $p$, see Sections 4 and 5. Proposition 5.1 asserts that if $f$ is monomial, then $R^{p}$ is independent of $p$ if and only if $f$ is a regular sequence. This motivates the following question.

Question B. Suppose that $f=\left(f_{1}, \ldots, f_{m}\right)$ is a sequence of germs of holomorphic functions at $0 \in \mathrm{C}^{n}$. Let $R^{p}(f)$ be the Bochner-Martinelli residue current of weight $p$. Is it true that $R^{p}(f)$ is independent of $p$ if and only if $f$ is a regular sequence? 
Question B could be asked also for ann $R^{p}(f)$ : is it true that ann $R^{p}(f)$ is independent of $p$ if and only if $f$ is a regular sequence?

Lemma 1.2 in [6] asserts that

$$
\sum_{\mathscr{I}=\left\{i_{1}, \ldots, i_{n}\right\} \subseteq\{1, \ldots, m\}} R_{\mathscr{I}}^{p}(f) \wedge d f_{i_{n}} \wedge \cdots \wedge d f_{i_{1}} /(2 \pi i)^{n}=e^{p}(f)[0],
$$

where $e^{p}(f)$ is a positive number; in fact each term in (1.5) is a positive current with support at the origin, see Lemma 3.1. Andersson [2] showed that $e^{(1, \ldots, 1)}(f)$ is the Hilbert-Samuel multiplicity of the ideal $a(f)$. In general $e^{p}(f)$ depends on $p$, see Example 4.4 , but it can also happen that $e^{p}(f)$ is independent of $p$ even if ann $R^{p}(f)$ and $R^{p}(f)$ vary with $p$, as shown in Example 5.5.

In general it is hard to compute $R^{p}(f)$, as well as ann $R^{p}(f)$ and $e^{p}(f)$. However, if the $f_{j}$ are monomials we can give an explicit description of $R^{p}(f)$ based on [23, Thm. 3.1]. For $A=\left\{a^{1}, \ldots, a^{m}\right\} \subseteq Z^{n}$, let $z^{A}$ denote the sequence of monomials $z^{a^{1}}, \ldots, z^{a^{m}}$, where $z^{a^{j}}=z_{1}^{a_{1}^{j}} \cdots z_{n}^{a_{n}^{j}}$ if $a^{j}=\left(a_{1}^{j}, \ldots, a_{n}^{j}\right)$. Moreover, for $p \in \mathrm{N}^{m}$, let $p A$ denote the set $p A=$ $\left\{p_{1} a^{1}, \ldots, p_{m} a^{m}\right\} \subseteq \mathrm{Z}^{n}$. Given a holomorphic function $g$ we will use the notation $\bar{\partial}[1 / g]$ for the value at $\lambda=0$ of $\bar{\partial}|g|^{2 \lambda} / g$, a priori defined for $\operatorname{Re} \lambda \gg 0$, and analogously by $[1 / g]$ we will mean $|g|^{2 \lambda} /\left.g\right|_{\lambda=0}$, that is, the principal value of $1 / \mathrm{g}$.

THEOREM C. Suppose that $z^{A}$ is a sequence of germs of holomorphic monomials at $0 \in C^{n}$, such that $V\left(z^{A}\right)=\{0\}$. Let $R^{p}\left(z^{A}\right)$ be the corresponding Bochner-Martinelli residue current of weight $p$. Then

$$
R_{\mathscr{I}}^{p}\left(z^{A}\right)=\operatorname{sgn}\left(A_{\mathscr{I}}\right) C_{\mathscr{I}} \bar{\partial}\left[\frac{1}{z_{1}^{\alpha_{1}^{\mathscr{Q}}}}\right] \wedge \cdots \wedge \bar{\partial}\left[\frac{1}{z_{n}^{\alpha_{n}^{\mathscr{Q}}}}\right] ;
$$

here $\operatorname{sgn}\left(A_{\mathscr{I}}\right)$ is the sign of the determinant of the matrix with rows $a^{i_{1}}, \ldots, a^{i_{n}}$, we have $C_{\mathscr{I}}>0$ if $\mathscr{I}$ is $p$-essential, and $C_{\mathscr{I}}=0$ otherwise, and $\left(\alpha_{1}^{\mathscr{I}}, \ldots\right.$, $\left.\alpha_{n}^{\mathscr{I}}\right)=\alpha^{\mathscr{I}}=\sum_{j \in \mathscr{I}} a^{j}$.

In particular, Theorem $\mathrm{C}$ implies that

$$
\text { ann } R^{p}\left(z^{A}\right)=\bigcap_{\mathscr{I} p \text {-essential }}\left(z_{1}^{\alpha_{1}^{\mathscr{S}}}, \ldots, z_{n}^{\alpha_{n}^{\mathscr{\Phi}}}\right) .
$$

In Section 2 we provide some background on Rees valuations, whereas the proof of Theorem A occupies Section 3. In Section 4 we focus on the case when $f$ is monomial; we prove Theorem $\mathrm{C}$ and compute the coefficients $C_{\mathscr{I}}$ 
in some special cases. Finally, in Section 5 we discuss Question B and some related questions.

\section{Rees valuations and essential multi-indices}

Let $f=\left(f_{1}, \ldots, f_{m}\right)$ be a sequence of germs of holomorphic functions at $0 \in \mathrm{C}^{n}$, such that $V(f)=\{0\}$. The Rees valuations of $\mathfrak{a}(f)$ are defined in terms of the normalized blowup $v: X^{+} \rightarrow\left(C^{n}, 0\right)$ of $a(f)$, see [13, Ch. II.7]. Since $V(\mathfrak{a})=\{0\}, v$ is an isomorphism outside $0 \in \mathrm{C}^{n}$ and $v^{-1}(0)$ is the union of finitely many prime divisors $E \subseteq X^{+}$. The Rees valuations of $a(f)$ are then the associated divisorial valuations $\operatorname{ord}_{E}$ on $\mathscr{O}_{0}^{n}: \operatorname{ord}_{E}(g)$ is the order of vanishing of $v^{*} g$ along $E$.

Let $\pi: X \rightarrow\left(C^{n}, 0\right)$ be a log-resolution of $a(f)$, see [16, Def. 9.1.12]. Then, in fact, a divisorial valuation $\operatorname{ord}_{E}$ is a Rees valuation of $a(f)$ if and only if the image of the prime divisor $E \subseteq \pi^{-1}(0)$ under the rational mapping $\Psi=\left[f_{1} \circ \pi: \ldots: f_{m} \circ \pi\right]: X \rightarrow \mathrm{CP}^{m-1}$ is of (maximal) dimension $n-1$, see $[20$, p. 332].

Consider a multi-index $\mathscr{I}=\left\{i_{1}, \ldots, i_{n}\right\} \subseteq\{1, \ldots, m\}$. Let $\pi_{\mathscr{I}}: \mathrm{CP}^{m-1} \backslash$ $W_{\mathscr{I}} \rightarrow \mathrm{CP}^{n-1}$, where $W_{\mathscr{I}}:=\left\{w_{i_{1}}=\ldots=w_{i_{n}}=0\right\} \subseteq \mathrm{CP}^{n}$, be the projection $\left[w_{1}: \ldots: w_{m}\right] \mapsto\left[w_{i_{1}}: \ldots: w_{i_{n}}\right]$. Following [15] we say that $\mathscr{I}$ is essential with respect to $E$ (and the sequence $f$ ) if $\Psi(E) \nsubseteq W_{\mathscr{I}}$ and the mapping $\pi_{\mathscr{I}} \circ \Psi: E \rightarrow \mathrm{CP}^{n-1}$ is surjective; in particular, $\operatorname{ord}_{E}\left(f_{i_{1}}\right)=\ldots=$ $\operatorname{ord}_{E}\left(f_{i_{n}}\right)=\operatorname{ord}_{E}(\mathfrak{a})$. Moreover we say that $\mathscr{I}$ is essential (with respect to $f$ ) if $\mathscr{I}$ is essential with respect to at least one exceptional prime. Furthermore we say that $\mathscr{I}$ is $p$-essential with respect to $E$ (and $f$ ) if $\mathscr{I}$ is essential with respect to the divisor $E$ and the sequence $f^{p}$, and that $\mathscr{I}$ is $p$-essential (with respect to $f$ ) if $\mathscr{I}$ is essential with respect to the sequence $f^{p}$.

Observe that if $\mathscr{I}$ is $p$-essential with respect to $E$, then $\operatorname{ord}_{E}$ must be a Rees valuation of $a\left(f^{p}\right)$. Conversely, if $\operatorname{ord}_{E}$ is a Rees valuation of $a\left(f^{p}\right)$, then there exists at least one multi-index $\mathscr{I}$, which is $p$-essential with respect to $E$. However, note that $\mathscr{I}$ can be $p$-essential with respect to more than one divisor $E$, and conversely there can be several multi-indices that are $p$-essential with respect to a given $E$.

Recall that the integral closure of $a \subseteq \mathscr{O}_{0}^{n}$ can be defined in terms of the Rees valuations of $\mathfrak{a}$. Indeed, $h \in \mathscr{O}_{0}^{n}$ is in $\overline{\mathfrak{a}}$ if and only if $\operatorname{ord}_{E}(h) \geq \operatorname{ord}_{E}(\mathfrak{a})$ for all Rees valuations ord $\operatorname{or}_{E}$ of $\mathfrak{a}$, see for example [16, Ex. 9.6.8].

Given a sequence $f$ and a multi-index $\mathscr{I}=\left\{i_{1}, \ldots, i_{n}\right\}$, let $f_{\mathscr{I}}$ denote the sequence $\left(f_{i_{1}}, \ldots, f_{i_{n}}\right)$. 


\section{Proof of Theorem A}

The proof of Theorem A is very much inspired by and based on (the proofs of) Theorems A and B in [15] and it also uses Andersson's construction of residue currents in [1]. The following result is Theorem B and Lemma 4.3 in [15].

Lemma 3.1. $R_{\mathscr{I}}(f) \not \equiv 0$ if and only if $\mathscr{I}$ is essential with respect to $f$. Moreover $R_{\mathscr{I}}(f) \wedge d f_{i_{n}} \wedge \cdots \wedge d f_{i_{1}} /(2 \pi i)^{n}$ is a positive current and its mass is strictly positive if and only if $\mathscr{I}$ is essential.

We first prove that $R_{\mathscr{I}}^{p}(f) \not \equiv 0$ precisely if $\mathscr{I}$ is $p$-essential. If $\mathscr{I}$ is not $p$-essential, then $R_{\mathscr{A}}\left(f^{p}\right)=0$ by Lemma 3.1, and hence in light of (1.2) $R_{\mathscr{I}}^{p}(f)=0$. For the converse, note that

$$
R_{\mathscr{I}}^{p}(f) \wedge d f_{i_{n}} \wedge \cdots \wedge d f_{i_{1}}=\frac{1}{p_{i_{1}} \ldots p_{i_{n}}} R_{\mathscr{I}}\left(f^{p}\right) \wedge d f_{i_{n}}^{p_{i_{n}}} \wedge \cdots \wedge d f_{i_{1}}^{p_{i_{1}}}
$$

by (1.2). Lemma 3.1 asserts that the right hand side of (3.1) is non-vanishing if $\mathscr{I}$ is essential with respect to $f^{p}$. Thus $R_{\mathscr{I}}^{p}(f) \not \equiv 0$ if $\mathscr{I}$ is $p$-essential.

The inclusion ann $R^{p}(f) \subseteq \mathfrak{a}(f)$ follows from Andersson's construction of global Bochner-Martinelli residue currents based on the Koszul complex in [1]. We provide (a sketch of) a proof for completeness.

We identify the sequence $f=\left(f_{1}, \ldots, f_{m}\right)$ with a holomorphic section of the dual bundle $V^{*}$ of a trivial rank $m$ vector bundle $V$ over some neighborhood $\mathcal{U}$ of $0 \in \mathrm{C}^{n}$, endowed with the trivial metric. If $\left\{e_{i}\right\}_{i=1}^{m}$ is a global holomorphic frame for $V$ and $\left\{e_{i}^{*}\right\}_{i=1}^{m}$ is the dual frame, we can write $f=\sum_{i=1}^{m} f_{i} e_{i}^{*}$. Let $s^{p}$ be the section $s^{p}=\sum_{i=1}^{m} \bar{f}_{i}\left|f_{i}\right|^{2\left(p_{i}-1\right)} e_{i}$, and let

$$
u^{p}=\sum_{\ell} \frac{s^{p} \wedge\left(\bar{\partial} s^{p}\right)^{\ell-1}}{\left|f^{p}\right|^{2 \ell}} .
$$

Then $u^{p}$ is a section of $\Lambda\left(V \oplus T_{0,1}^{*}(\mathscr{U})\right)$ (where $\left.e_{j} \wedge d \bar{z}_{i}=-d \bar{z}_{i} \wedge e_{j}\right)$, that is clearly well-defined and smooth outside $V(f)=\{0\}$, and moreover $\bar{\partial}\left|f^{p}\right|^{2 \lambda} \wedge u^{p}$, has an analytic continuation as a current to $\operatorname{Re} \lambda>-\epsilon$, see [1]. Note that the $e_{i_{n}} \wedge \cdots \wedge e_{i_{1}}$-coefficient of $R\left(u^{p}\right):=\left.\bar{\partial}|f|^{2 \lambda} \wedge u^{p}\right|_{\lambda=0}$ is just the current $R_{\mathscr{I}}^{p}(f)$, and thus in particular, ann $R\left(u^{p}\right)=$ ann $R^{p}(f)$. Let $\nabla=\delta_{f}-\bar{\partial}: \Lambda\left(V \oplus T_{0,1}^{*}(\mathscr{U})\right) \rightarrow \Lambda\left(V \oplus T_{0,1}^{*}(\mathscr{U})\right)$; here $\delta_{f}$ denotes interior multiplication by $f$. Observe that $\nabla u^{p}=1$ outside $V(f)$. In [1] it was proved if $u$ is any section of $\Lambda\left(V \oplus T_{0,1}^{*}(\mathscr{U})\right)$ that is smooth and satisfies $\nabla u=1$ outside $V(f)$, then the corresponding current $R(u):=\left.\bar{\partial}|f|^{2 \lambda} \wedge u\right|_{\lambda=0}$ satisfies that ann $R(u) \subseteq \mathfrak{a}(f)$. We conclude that ann $R^{p}(f) \subseteq \mathfrak{a}(f)$.

Given a sequence of germs $g_{1}, \ldots, g_{n} \in \mathscr{O}_{0}^{n}$, let $\operatorname{Jac}(g)$ denote the Jacobian determinant $\operatorname{Jac}(g)=\left|\frac{\partial g_{i}}{\partial z_{j}}\right|_{1 \leq i, j \leq n}$. Note that $d f_{i_{n}} \wedge \cdots \wedge d f_{i_{1}}= \pm \operatorname{Jac}\left(f_{I}\right) d z_{n} \wedge$ 
$\cdots \wedge d z_{1}$. Thus in light of (3.1) and Lemma 3.1, $\operatorname{Jac}\left(f_{\mathscr{I}}\right) \in$ ann $R_{\mathscr{I}}^{p}(f)$ if and only if $R_{\mathscr{I}}^{p}(f) \equiv 0$. Given this we can show that ann $R^{p}(f)=\mathfrak{a}(f)$ implies that $a(f)$ is a complete intersection ideal by following the proof of Theorem A in [15, Section 5].

It remains to prove that the right inclusion in (1.3) is strict when $n \geq 2$. Given a multi-index $\mathscr{I}=\left\{i_{1}, \ldots, i_{n}\right\}$, let $P(\mathscr{I})=\sum_{j=1}^{n} \frac{1}{p_{i_{j}}}$. Pick two multi-indices $\mathscr{I}$ and $\mathscr{J}$, such that $P(\mathscr{I}) \geq P(\mathscr{J})$. We claim that then $R_{\mathscr{J}}^{p}(f) \wedge d f_{i_{n}} \wedge \cdots \wedge d f_{i_{1}}$ either vanishes or is a positive pointmass at the origin.

Let $\pi: X \rightarrow\left(C^{n}, 0\right)$ be a log-resolution of $a\left(f^{p}\right)$. Then $R_{\mathscr{J}}\left(f^{p}\right)$ is the push-forward of a current $\tilde{R}$ on $X$, which has support on the exceptional primes with respect to whom $\mathscr{J}$ is essential. More precisely, $\tilde{R}$ can be decomposed as $\tilde{R}=\sum \tilde{R}_{E}$, where the sum is over the exceptional primes $E \subseteq X$, such that $\mathscr{J}$ is essential with respect to $E$, and $\tilde{R}_{E}$ has support on $E$, see [15, Section 6].

Let $E_{1}$ be an exceptional prime, such that $\mathscr{J}$ is essential with respect to $E_{1}$. Then we can choose local coordinates $\sigma$ on $X$, so that $E_{1}=\left\{\sigma_{1}=0\right\}$ and $\tilde{R}_{E_{1}}$ is of the form $\bar{\partial}\left[1 / \sigma_{1}^{n a_{1}}\right] \wedge\left[1 /\left(\sigma_{2}^{n a_{2}} \ldots \sigma_{n}^{n a_{n}}\right)\right] \wedge \beta$, where $\beta$ is a smooth form and $a_{j}=\operatorname{ord}_{E_{j}}\left(f^{p}\right)$, where $E_{j}=\left\{\sigma_{j}=0\right\}$. Observe that for $1 \leq \ell \leq m$, $\pi^{*} f_{\ell}^{p_{\ell}}$ is divisible by $\sigma_{j}^{a_{j}}$ and so $\pi^{*} f_{\ell}$ is divisible by $\sigma_{j}^{\left\lceil a_{j} / p_{\ell}\right\rceil}$. It follows that

$$
\pi^{*}\left(f_{j_{1}}^{p_{j_{1}}-1} \ldots f_{j_{n}}^{p_{j_{n}}-1}\right) \tilde{R}_{E_{1}}=\bar{\partial}\left[1 / \sigma_{1}^{b_{1}}\right] \wedge\left[1 /\left(\sigma_{2}^{b_{2}} \ldots \sigma_{n}^{b_{n}}\right)\right] \wedge \beta,
$$

where $b_{j} \leq a_{j} P(\mathscr{J})$. A computation following [15, p. 2130] yields that

$$
\pi^{*}\left(d f_{i_{n}} \wedge \cdots \wedge d f_{i_{1}}\right)=\sigma_{1}^{c_{1}-1}\left(\sigma_{2}^{c_{2}} \ldots \sigma_{n}^{c_{n}} \gamma+\sigma_{1} \delta\right) d \sigma_{1} \wedge \cdots \wedge d \sigma_{n},
$$

where $c_{j} \geq a_{j} P(\mathscr{I})$ and $\gamma$ and $\delta$ are holomorphic functions. Since, by assumption, $P(\mathscr{I}) \geq P(\mathscr{J}), \pi^{*}\left(f_{j_{1}}^{p_{j_{1}}-1} \ldots f_{j_{n}}^{p_{j_{n}}-1}\right) \tilde{R}_{E_{1}} \wedge \pi^{*}\left(d f_{i_{n}} \wedge \cdots \wedge d f_{i_{1}}\right)$ is of the form $\bar{\partial}\left[1 / \sigma_{1}\right] \wedge d \sigma_{1} \wedge \tilde{\beta}=2 \pi i\left[E_{1}\right] \wedge \tilde{\beta}$, where $\tilde{\beta}$ is a smooth form. Hence

$$
\begin{aligned}
R_{\mathscr{J}}^{p}(f) \wedge d f_{i_{n}} & \wedge \cdots \wedge d f_{i_{1}} \\
& =\sum_{E} \pi_{*}\left(\pi^{*}\left(f_{j_{1}}^{p_{j_{1}}-1} \ldots f_{j_{n}}^{p_{j_{n}}-1}\right) \tilde{R}_{E} \wedge \pi^{*}\left(d f_{i_{n}} \wedge \cdots \wedge d f_{i_{1}}\right)\right)
\end{aligned}
$$

is a non-negative point mass at 0 and the claim is proved.

Now pick a $p$-essential multi-index $\mathscr{I}$, for which $P(\mathscr{I})=\max _{\mathscr{J} p \text {-essential }} P(\mathscr{J})$. Then the non-vanishing entries of $R^{p}(f) \wedge d f_{i_{n}} \wedge \cdots \wedge d f_{i_{1}}$ are just pointmasses at the origin; in particular, $\operatorname{Jac}\left(f_{\mathscr{I}}\right) \mathfrak{m} \subseteq$ ann $R^{p}(f)$, where $\mathfrak{m}$ denotes the maximal ideal in $\mathscr{O}_{0}^{n}$. Let $E$ be an exceptional prime, such that $\mathscr{I}$ is $p$ essential with respect to $E$. A direct computation gives that $\operatorname{ord}_{E}\left(d f_{i_{1}}^{p_{i_{1}}} \wedge \cdots \wedge\right.$ 
$\left.d f_{i_{n}}^{p_{i_{n}}}\right)=n \operatorname{ord}_{E}\left(f^{p}\right)-1$ and $\operatorname{ord}_{E}\left(d z_{1} \wedge \cdots \wedge d z_{n}\right) \geq \sum_{i=1}^{n} \operatorname{ord}_{E}\left(z_{i}\right)-1$. Note that $\operatorname{ord}_{E}\left(z_{k}\right) \geq 1$ for $1 \leq k \leq n$. Since $d f_{i_{1}}^{p_{i_{1}}} \wedge \cdots \wedge d f_{i_{n}}^{p_{i_{n}}}=$ $p_{i_{1}} \ldots p_{i_{n}} f_{i_{1}}^{p_{i_{1}}-1} \ldots f_{i_{n}}^{p_{i_{n}}-1} \operatorname{Jac}\left(f_{\mathscr{I}}\right) d z_{1} \wedge \cdots \wedge d z_{n}$ it follows that

$$
\begin{aligned}
\operatorname{ord}_{E}\left(z_{k} f_{i_{1}}^{p_{i_{1}}-1} \ldots f_{i_{n}}^{p_{i_{n}}-1} \operatorname{Jac}\left(f_{\mathscr{I}}\right)\right) & \leq n \operatorname{ord}_{E}\left(f^{p}\right)-n+1 \\
& =\operatorname{ord}_{E} \overline{\mathfrak{a}\left(f^{p}\right)^{n}}-n+1
\end{aligned}
$$

for $1 \leq k \leq n$. Here we have used that $\bar{a}$ is the set of all $h \in \mathcal{O}_{0}^{n}$, that satisfy $\operatorname{ord}_{E}(h) \geq \operatorname{ord}_{E}(\mathfrak{a})$ for all Rees valuations $\operatorname{ord}_{E}$ of a, see Section 2. Hence, if $n \geq 2$, there are elements, for example $z_{k} \operatorname{Jac}\left(f_{\mathscr{I}}\right)$, in ann $R^{p}(f)$ that are not in $\overline{\mathfrak{a}\left(f^{p}\right)^{n}}:\left(f_{i_{1}}^{p_{i_{1}}-1} \ldots f_{i_{n}}^{p_{i_{n}}-1}\right)$. This proves that the first inclusion in (1.3) is strict for $n \geq 2$ and concludes the proof of Theorem A.

\section{The monomial case}

Let $z^{A}=\left(z^{a^{1}}, \ldots, z^{a^{m}}\right)$ be a sequence of germs of monomials in $\mathscr{O}_{0}^{n}$. Recall that the Newton polyhedron $\mathrm{NP}(A)$ is defined as the convex hull in $\mathrm{R}^{n}$ of the set of exponents of monomials in $\mathfrak{a}\left(z^{A}\right)$. The Rees valuations of $\mathfrak{a}\left(z^{A}\right)$ are monomial and in one-to-one correspondence with the compact facets (faces of dimension $n-1)$ of $\mathrm{NP}(A)$. More precisely, the facet $\tau$ with normal vector $\rho=\left(\rho_{1}, \ldots, \rho_{n}\right)$ corresponds to the monomial valuation $\operatorname{ord}_{\tau}\left(z_{1}^{a_{1}} \ldots z_{n}^{a_{n}}\right)=$ $\rho_{1} a_{1}+\cdots+\rho_{n} a_{n}$, see for example [14, Thm. 10.3.5]. Given a multi-index $\mathscr{I}=\left\{i_{1}, \ldots, i_{n}\right\}$, let $A_{\mathscr{I}}$ denote the set $\left\{a^{i_{1}}, \ldots, a^{i_{n}}\right\} \subseteq A$ so that $z^{A_{\mathscr{G}}}$ is the sequence $z^{a^{i_{1}}}, \ldots, z^{a^{i_{n}}}$. Moreover, let $\operatorname{det}\left(A_{\mathscr{I}}\right)$ denote the determinant of the matrix with rows $a^{i_{1}}, \ldots, a^{i_{n}}$. It follows that a multi-index $\mathscr{I}$ is essential with respect to $E_{\tau}$ precisely if $A_{\mathscr{I}}$ is contained in $\tau$ and $\operatorname{det}\left(A_{\mathscr{I}}\right) \neq 0$; here $E_{\tau}$ denotes the exceptional prime associated with $\tau$. This means that $\mathscr{I}$ is $p$ essential if and only if $p A_{\mathscr{I}}:=\left\{p_{i_{1}} a^{i_{1}}, \ldots, p_{i_{n}} a^{i_{n}}\right\}$ is contained in a facet of $\mathrm{NP}(p A)$ and $\operatorname{det}\left(A_{\mathscr{I}}\right) \neq 0$.

Observe that if $V\left(z^{A}\right)=\{0\}$, then $z^{A}$ is regular precisely if $m=n$ and $z^{a^{j}}$ is of the form $z_{j}^{b_{j}}$ (possibly after reordering the variables). Moreover, recall that the integral closure of $a\left(z^{A}\right)$ is the monomial ideal generated by monomials with exponents in $\mathrm{NP}(A)$, see for example [20].

Let us illustrate Theorem $\mathrm{C}$ with some examples.

EXAMPLE 4.1. Let $z^{A}$ be the sequence of monomials $z^{A}=\left(z^{a^{1}}, \ldots, z^{a^{4}}\right)=$ $\left(z_{1}^{5}, z_{1}^{4} z_{2}, z_{1}^{2} z_{2}^{2}, z_{2}^{3}\right)$. Then $\mathrm{NP}(A)$ has just one compact facet and so $\mathfrak{a}\left(z^{A}\right)$ has exactly one Rees valuation, which is the monomial valuation $\operatorname{ord}_{E}$ given by $\operatorname{ord}_{E}\left(z_{1}^{b_{1}} z_{2}^{b_{2}}\right)=3 b_{1}+5 b_{2}$. Moreover the only essential multi-index with respect to $z^{A}$ is $\{1,4\}$ and so Theorem $\mathrm{C}$ asserts that $R\left(z^{A}\right)=R^{p}\left(z^{A}\right)$, where $p=$ 


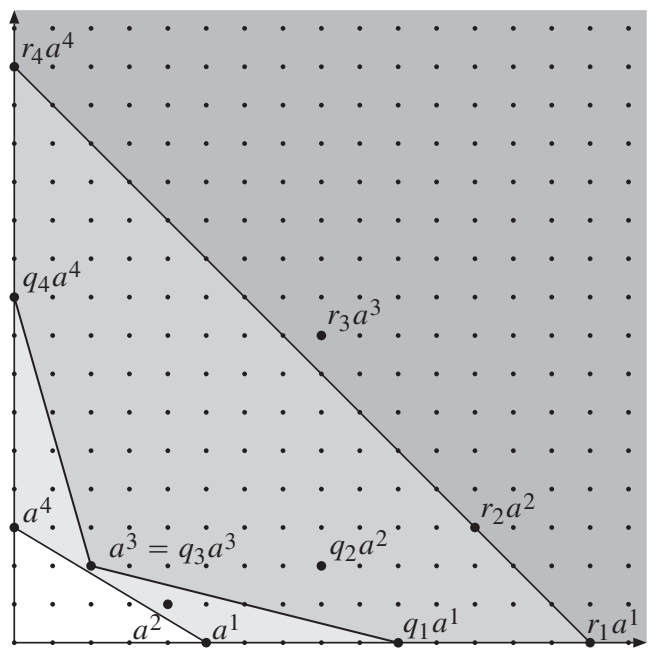

Figure 1. The Newton polytopes of the sequences $z^{A}$ (light grey), $z^{q A}$ (medium grey), and $z^{r A}$ (dark grey) in Example 4.1.

$(1,1,1,1)$, has one non-vanishing entry $R_{\{1,4\}}\left(z^{A}\right)=C_{\{1,4\}} \bar{\partial}\left[\frac{1}{z_{1}^{5}}\right] \wedge \bar{\partial}\left[\frac{1}{z_{2}^{3}}\right]$ and ann $R\left(z^{A}\right)=\left(z_{1}^{5}, z_{2}^{3}\right)$.

Let $q=(2,2,1,3)$. Then $\mathrm{NP}(q A)$ has two compact facets, so that $\mathfrak{a}\left(z^{q A}\right)=$ $\left(z_{1}^{10}, z_{1}^{8} z_{2}^{2}, z_{1}^{2} z_{2}^{2}, z_{2}^{9}\right)$ has two Rees valuations: $\operatorname{ord}_{E_{1}}\left(z_{1}^{b_{1}} z_{2}^{b_{2}}\right)=b_{1}+4 b_{2}$ and $\operatorname{ord}_{E_{2}}\left(z_{1}^{b_{1}} z_{2}^{b_{2}}\right)=7 b_{1}+2 b_{2}$. Moreover there are two $q$-essential multi-indices, $\{1,3\}$ and $\{3,4\}$, corresponding to $E_{1}$ and $E_{2}$, respectively. It follows from Theorem $\mathrm{C}$ that ann $R^{q}\left(z^{A}\right)=\left(z_{1}^{7}, z_{2}^{2}\right) \cap\left(z_{1}^{2}, z_{2}^{5}\right)=\left(z_{1}^{7}, z_{1}^{2} z_{2}^{2}, z_{2}^{5}\right)$. Note that ann $R^{p} \nsubseteq$ ann $R^{q}$ and ann $R^{q} \nsubseteq$ ann $R^{p}$, which illustrates that in general no relation between the weights $p$ and $q$ is reflected in the relation between ann $R^{p}\left(z^{A}\right)$ and ann $R^{q}\left(z^{A}\right)$. One can check that by varying the weight $p$ one gets all together 9 different annihilator ideals. Let us consider one more example. Let $r=(3,3,4,5)$. Then $\mathrm{NP}(r A)$ has one compact facet, so that $\mathfrak{a}\left(z^{r A}\right)$ has one Rees valuation. However, there are three $r$-essential multiindices, $\{1,2\},\{1,4\}$, and $\{2,4\}$, and ann $R^{r}\left(z^{A}\right)=\left(z_{1}^{9}, z_{2}\right) \cap\left(z_{1}^{5}, z_{2}^{3}\right) \cap\left(z_{1}^{4}, z_{2}^{4}\right)$. In Figure 1 we have drawn $\mathrm{NP}(p A)$ and also marked the elements in $p A$, for the weights $p, q$, and $r$.

Note that $z^{\left(q_{1}-1\right) a^{1}} z^{\left(q_{3}-1\right) a^{3}}=z_{1}^{5}$ and $z^{\left(q_{3}-1\right) a^{3}} z^{\left(q_{4}-1\right) a^{4}}=z_{2}^{6}$. It follows that for the weight $q$ the leftmost ideal in (1.3) is given by $\left(z_{1}^{15}, z_{1}^{11} z_{2}, z_{1}^{7} z_{2}^{2}, z_{1}^{3} z_{2}^{3}\right.$, $\left.z_{1}^{2} z_{2}^{5}, z_{1} z_{2}^{9}, z_{2}^{12}\right)$ and so one sees directly that the left inclusion in (1.3) is strict in this case. In Figure 2 the three ideals in (1.3) are depicted for weights $p$, $q$, and $r$. Note that ann $R^{p}\left(z^{A}\right)$ is strictly included in $a\left(z^{A}\right)$ is all three cases. Also note that $\overline{\mathfrak{a}\left(z^{A}\right)^{2}} \nsubseteq$ ann $R^{r}\left(z^{A}\right)$, which shows that it is not true in general that $\overline{a(f)^{n}} \subseteq$ ann $R^{p}(f)$. 

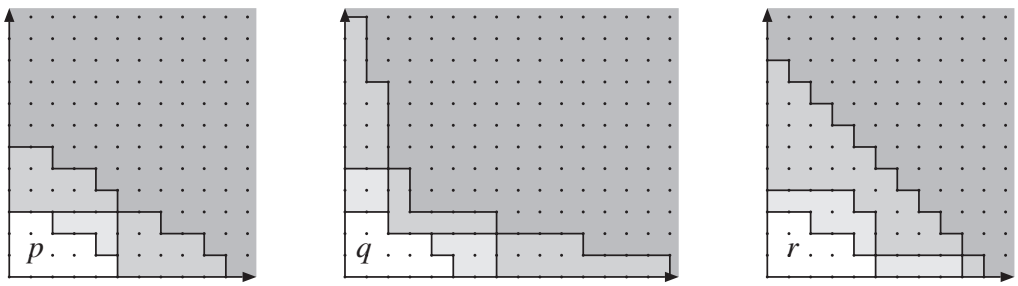

Figure 2. The exponent sets of the ideals $a\left(z^{A}\right)$ (light grey), ann $R^{p}\left(z^{A}\right)$ (medium grey) and $\bigcap \overline{\left(z^{A}\right)^{2}}:\left(z^{\left(p_{i_{1}}-1\right) a^{i_{1}}} z^{\left(p_{i_{2}}-1\right) a^{i_{2}}}\right)$ (dark grey) for weights $p, q$, and $r$ in Example 4.1.

EXAMPLE 4.2. Let $z^{A}=\left(z, z^{2}\right)$. Then $a\left(z^{A}\right)$ is just the maximal ideal $\mathfrak{m} \subseteq \mathscr{O}_{0}^{1}$. Note that since $n=1$ there is a unique Rees valuation associated with $\mathfrak{a}\left(z^{A}\right)$, namely the order of vanishing at the origin. For $j \in \mathrm{N}$, let $p^{j}=(j, 1)$. Then $R\left(z^{A}\right)=R^{p^{1}}\left(z^{A}\right)=(\bar{\partial}[1 / z], 0), R^{p^{2}}\left(z^{A}\right)=\left(\bar{\partial}[1 / z], \bar{\partial}\left[1 / z^{2}\right]\right)$, and $R^{p^{j}}\left(z^{A}\right)=\left(0, \bar{\partial}\left[1 / z^{2}\right]\right)$ for $j \geq 3$. It follows that ann $R=\mathfrak{m}$, whereas ann $R^{p^{j}}=\mathfrak{m}^{2}$ for $j \geq 2$.

Example 4.2 shows that in general $R^{p}(f)$, as well as ann $R^{p}(f)$, depends in an essential way on the particular sequence $f$ and not only on the ideal $a(f)$. Theorem A in [15] asserts that ann $R(f)=a(f)$ if and only if $a(f)$ is a complete intersection ideal. Theorem A says that the only if-direction of this statement holds for any $p$, whereas Example 4.2 shows that the if-direction fails in general. Moreover, in the monomial case $R(f)$ only depends on $a(f)$ and not on the particular sequence $f$. Question D in [15] asks whether it is always true (as long as $V(f)=\{0\}$ ) that ann $R(f)$ only depends on $a(f)$.

\subsection{Proof of Theorem $C$}

Theorem 3.1 in [23] states that if $\mathscr{I}$ is essential with respect to $z^{A}$, then $R_{\mathscr{I}}(f)$ is of the form (1.6), where $C_{\mathscr{I}}$ is a nonzero constant. Thus, using (1.2) and (1.4), we conclude that the entries of $R^{p}(f)$ are of the form (1.6).

Assume that $\mathscr{I}$ is $p$-essential. Then by Lemma 3.1, (3.1) times $1 /(2 \pi i)^{n}$ has strictly positive mass. Note that $d z^{a^{i_{n}}} \wedge \cdots \wedge d z^{a^{i_{1}}}=\operatorname{det}\left(A_{\mathscr{S}}\right) z_{1}^{\alpha_{1}^{\mathscr{Q}}-1} \cdots z_{n}^{\alpha_{n}^{\mathscr{q}}-1}$ $d z_{n} \wedge \cdots \wedge d z_{1}$. Since $\bar{\partial}\left[\frac{1}{z}\right] \wedge d z=2 \pi i[0]$, it follows that the left hand side of (3.1) is equal to $(2 \pi i)^{n} C_{\mathscr{I}}\left|\operatorname{det}\left(A_{\mathscr{I}}\right)\right|[0]$, and so $C_{\mathscr{I}} \geq 0$.

\subsection{The coefficients $C_{\mathscr{I}}$}

Given a sequence of monomials $z^{A}$ one can find a log-resolution $X_{A} \rightarrow\left(C^{n}, 0\right)$ of $a\left(z^{A}\right)$, where $X_{A}$ is a toric variety constructed from (the normal fan of) $\mathrm{NP}(A)$, see [7, p. 82]. In [23] we computed $R\left(z^{A}\right)$ as the push-forward of a certain current on $X_{A}$. Assume that $\mathscr{I}$ is essential with respect to $E_{\tau}$, where $\tau$ is a facet of $\operatorname{NP}(A)$. According to [23, p. 381], the coefficient $C_{\mathscr{I}}$ is of the 
form $C_{\mathscr{I}}= \pm \frac{1}{(2 \pi i)^{n-1}}(n-1) ! D I$, where $I$ is an integral of the form

$$
I=\int \frac{\prod_{j=1}^{n-1}\left|t_{j}\right|^{2\left(c_{j 1}+\cdots+c_{j n}-1\right)}}{\sum_{k=1}^{\ell} \prod_{j=1}^{n-1}\left|t_{j}\right|^{2 c_{j k}}} d \bar{t}_{1} \wedge \cdots \wedge d \bar{t}_{n-1} \wedge d t_{n-1} \wedge \cdots \wedge d t_{1},
$$

for some $n \leq \ell \leq m$ and $\left\{c_{j k}\right\}_{1 \leq j \leq n-1,1 \leq k \leq \ell}$, and $D$ is the determinant of the matrix with entries $\left\{d_{j k}\right\}_{1 \leq j, k \leq n}$, where $d_{j k}=c_{j k}$ if $j \leq n-1$ and $d_{n k}=1$. The terms in the denominator correspond to the $a^{j} \in A$ that lie in $\tau$; in particular, $C_{\mathscr{I}}$ depends only on $\tau \cap A$. (Assuming that $\mathscr{I}=\{1, \ldots, n\}$ and that $\left\{a^{1}, \ldots, a^{\ell}\right\}$ are the exponents in $\tau$, then, in the terminology of [23], $c_{j k}=\rho_{j} \cdot\left(b_{k}-a_{0}\right)$.) In general the integral $I$ is hard to compute; compare to (5.1).

Assume that $\ell=n$ and that $c_{j k}=0$ unless $j=k$, possibly after rearranging the variables $t_{j}$. Then

$$
I=\int \frac{\prod_{j=1}^{n-1}\left|t_{j}\right|^{2\left(c_{j}-1\right)}}{\left(1+\sum_{j=1}^{n-1}\left|t_{j}\right|^{2 c_{j}}\right)^{n}} d \bar{t}_{1} \wedge \cdots \wedge d \bar{t}_{n-1} \wedge d t_{n-1} \wedge \cdots \wedge d t_{1},
$$

where $c_{j}$ just denotes $c_{j j}$. A direct computation gives that

$$
\int \frac{|s|^{2(N-1)}}{\left(1+|s|^{2 N}\right)^{p}} d \bar{s} \wedge d s=2 \pi i \frac{1}{p-1} \frac{1}{N},
$$

which implies that $I=\frac{(2 \pi i)^{n-1}}{(n-1) !} \frac{1}{c_{1} \cdots c_{n-1}}$. Moreover $D=c_{1} \cdots c_{n-1}$, and since $C_{\mathscr{I}} \geq 0$, we conclude that $C_{\mathscr{I}}=1$.

The assumption that $\ell=n$ is satisfied precisely if $\mathscr{I}$ is the unique multiindex that is essential with respect to a certain Rees valuation. The assumption that $c_{j k}=0$ for $j \neq k$ is for example satisfied if the normal fan of $\mathrm{NP}(A)$ is regular, see [11]. It is also satisfied if $n=2$.

Given a facet $\tau$ of $\operatorname{NP}(A)$, let $\operatorname{det}(\tau)$ be the normalized volume, that is, $n$ ! times the Euclidean volume, of the convex hull of $\tau$ and the origin in $\mathrm{R}^{n}$. If $\tau$ is simplicial with vertices $b^{1}, \ldots, b^{n}$, then $\operatorname{det}(\tau)$ is just (the absolute value of) the determinant of the matrix with rows $b^{1}, \ldots, b^{n}$. For $n=2$ we have the following description of the coefficients $C_{\mathscr{I}}$ :

$$
\sum_{A_{\mathscr{S}} \subseteq \tau}\left|\operatorname{det}\left(A_{\mathscr{I}}\right)\right| C_{\mathscr{I}}=\operatorname{det}(\tau) .
$$

To prove this, recall that if $V\left(z^{A}\right)=\{0\}$, then the Hilbert-Samuel multiplicity $e\left(z^{A}\right)$ of $\mathfrak{a}\left(z^{A}\right)$ equals the normalized volume $\operatorname{Vol}\left(\mathrm{R}_{+}^{n} \backslash \mathrm{NP}(A)\right)$ of the complement in $\mathrm{R}_{+}^{n}$ of $\mathrm{NP}(A)$, see for example [21]. Observe that $\operatorname{Vol}\left(\mathrm{R}_{+}^{n} \backslash \mathrm{NP}(A)\right)=$ 
$\sum \operatorname{det}(\tau)$, where the sum runs over the facets $\tau$ of $\operatorname{NP}(A)$. Now (4.1) follows in light of (1.5) and the fact that if $\mathscr{I}$ is essential with respect to $E_{\tau}$, then $C_{\mathscr{I}}$ depends only on $a^{j} \in A \cap \tau$.

Question 4.3. Does (4.1) hold also when $n>2$ ?

ExAmple 4.4. Let $z^{A}$ and $p, q$, and $r$ be as in Example 4.1, and let $s=$ $(2,1,1,2)$. From [2] we know that $e^{p}\left(z^{A}\right)$ is the Hilbert-Samuel multiplicity of $a\left(z^{A}\right)$. Since there is only one essential multi-index with respect to $z^{A}$ we can also compute this directly from (4.1). Indeed $C_{\{1,4\}}=1$ and so $e^{p}\left(z^{A}\right)=$ $\left|\operatorname{det}\left(A_{\{1,4\}}\right)\right|=15$.

Moreover, recall that $a\left(z^{q A}\right)$ has two Rees valuations and that there is one $q$-essential multi-index associated with each divisor: $\{1,3\}$ and $\{3,4\}$. Hence $C_{\{1,3\}}=C_{\{3,4\}}=1$ and so $e^{q}\left(z^{A}\right)=\left|\operatorname{det}\left(A_{\{1,3\}}\right)\right|+\left|\operatorname{det}\left(A_{\{3,4\}}\right)\right|=10+6=$ 16 , that is, the normalized area of the convex hull of $a^{1}=(5,0), a^{3}=(2,2)$, and $a^{4}=(0,3)$. Similarly $a\left(z^{s A}\right)$ has three Rees valuations and there is one $s$-essential multi-index for each valuation; it follows that $e^{s}\left(z^{A}\right)=17$, see Figure 3.
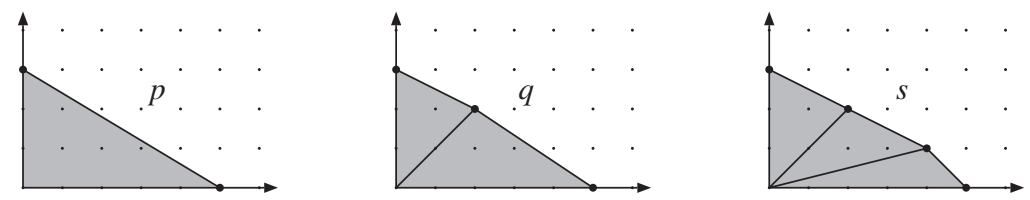

Figure 3. The multiplicities $e^{p}\left(z^{A}\right), e^{q}\left(z^{A}\right)$, and $e^{s}\left(z^{A}\right)$ in Example 4.4.

Finally $a\left(z^{r A}\right)$ has one Rees valuation, but there are three $r$-essential multiindices. From (4.1) we know that $C_{\{1,2\}}\left|\operatorname{det}\left(A_{\{1,2\}}\right)\right|+C_{\{1,4\}}\left|\operatorname{det}\left(A_{\{1,4\}}\right)\right|+$ $C_{\{2,4\}}\left|\operatorname{det}\left(A_{\{2,4\}}\right)\right|=\left|\operatorname{det}\left(A_{\{1,4\}}\right)\right|$, which means $5 C_{\{1,2\}}+15 C_{\{1,4\}}+12 C_{\{2,4\}}=$ 15. However, we cannot say more; in particular, we cannot determine $e^{r}\left(z^{A}\right)$.

\section{Discussion of Question B}

Theorem C allows us to give an affirmative answer to Question B in the monomial case. Recall that if $a(f)$ is a complete intersection ideal, then $a(f)$ is, in fact, generated by $n$ of the $f_{j}$. This follows for example from Nakayama's Lemma.

Proposition 5.1. Suppose that $z^{A}=\left(z^{a^{j}}\right)_{j=1}^{m}$ is a sequence of germs of holomorphic monomials at $0 \in \mathrm{C}^{n}$, such that $V\left(z^{A}\right)=\{0\}$. Then $R^{p}\left(z^{A}\right)$ is independent of $p$ if and only if $z^{A}$ is a regular sequence.

Moreover, ann $R^{p}\left(z^{A}\right)$ is independent of $p$ if and only if for each $\mathscr{I}=$ $\left\{i_{1}, \ldots, i_{n}\right\} \subseteq\{1, \ldots, m\}$, either $z^{A_{\mathscr{I}}}$ generates $a\left(z^{A}\right)$ or $\operatorname{det}\left(A_{\mathscr{I}}\right)=0$. 
Note that the condition that either $z^{A_{\mathscr{J}}}$ generates $\mathfrak{a}\left(z^{A}\right)$ or $\operatorname{det}\left(A_{\mathscr{I}}\right)=0$ is equivalent to that $\mathfrak{a}\left(z^{A}\right)$ is a complete intersection ideal, generated by say $z_{1}^{b_{1}}, \ldots, z_{n}^{b_{n}}$, and that moreover, for $1 \leq j \leq m, z^{a^{j}}$ is equal to $z_{k}^{b_{k}}$ for some $1 \leq k \leq n$.

Proof. First, note that the if-directions of the statements in Proposition 5.1 follow immediately from Theorem $\mathrm{C}$. Thus we need to prove the only ifdirections.

Let $\mathscr{I}$ be a multi-index defined by that $z^{a_{i j}}$ is of the form $z_{j}^{b_{j}}$, where $b_{j}$ is the smallest number such that $z_{j}^{b_{j}}$ is among the entries of $z^{A}$. Without loss of generality we may assume that $\mathscr{I}=\{1, \ldots, n\}$. Choose $p \in \mathrm{N}^{m}$, so that $p_{i}=1$ if $i \leq n$ and $p_{i} \gg 1$ otherwise. Then $\mathscr{I}$ is the unique $p$-essential multi-index.

Assume that $m>n$ and choose $j$, such that $n<j \leq m$. Moreover, choose $q \in \mathrm{N}^{m}$ such that $a^{j}$ lies in the one of the compact facets of $\mathrm{NP}(q A)$. For example, let $q$ be defined by $q_{i}=\left|a^{1}\right|+\cdots+\left|a^{i-1}\right|+\left|a^{i+1}\right|+\cdots+\left|a^{m}\right|$, where $\left|a^{\ell}\right|=a_{1}^{\ell}+\cdots+a_{n}^{\ell}$. Then $j$ is contained in a $q$-essential multi-index, say $\mathscr{J}$. It follows that $R_{\mathscr{J}}^{q}\left(z^{A}\right) \neq 0$, whereas $R_{\mathscr{J}}^{p}\left(z^{A}\right)=0$. Hence $R^{p}\left(z^{A}\right) \neq R^{q}\left(z^{A}\right)$ and we have proved the first part of Proposition 5.1.

Next, assume that there is an $a^{j} \in A$ such that $z^{a_{j}}$ is not equal to any of $z_{1}^{b_{1}}, \ldots, z_{n}^{b_{n}}$. Since $V\left(z^{A}\right)=\{0\}$, at least one of the entries of $a^{j}$ is positive, say $a_{k}^{j}>0$. Let $\mathscr{J}=\{1, \ldots, k-1, k+1, \ldots, n, j\}$. Then $\operatorname{det}\left(A_{\mathscr{J}}\right) \neq 0$, which means that we can find a weight $q$ such that $\mathscr{J}$ is $q$-essential; for instance we can take $q$ as above. By assumption, $a_{k}^{j}>b_{k}$ or $a_{i}^{j}>0$ for some $i \neq k$. In both cases, for some $\ell$, the $\ell$ th entry of $\sum_{j \in \mathscr{J}} a^{j}$ is strictly larger than the $\ell$ th entry of $\sum_{j \in \mathscr{I}} a^{j}$ and thus ann $R_{\mathscr{J}}^{q}\left(z^{A}\right) \nsupseteq$ ann $R_{\mathscr{I}}^{p}\left(z^{A}\right)$. This proves the second part of Proposition 5.1.

Observe that a necessary condition for Question B to be true would be that the set of $p$-essential multi-indices is independent of $p$ if and only if $f$ is a regular sequence. As we saw in the above proof this is true if $f$ is monomial, but we do not know if it holds in general. When $f$ is monomial, the essential multi-indices are rather special. For example, a multi-index can be essential with respect to at most one Rees valuation, which is not the case in general. Indeed, if $m=n$, then $\mathscr{I}=\{1, \ldots, n\}$ is essential with respect to all Rees valuations (and there can be more than one Rees valuation). The following example illustrates another phenomenon, which does not occur in the monomial case.

EXAMPLE 5.2. Let $f=\left(z_{1}^{4}-z_{2}^{4}, z_{1}^{2} z_{2}, z_{1} z_{2}^{2}\right)$. Then $\mathfrak{a}(f)$ has three Rees valuations, namely the monomial valuations $\operatorname{ord}_{E_{1}}\left(z_{1}^{b_{1}} z_{2}^{b_{2}}\right)=b_{1}+b_{2}$, $\operatorname{ord}_{E_{2}}\left(z_{1}^{b_{1}} z_{2}^{b_{2}}\right)=2 b_{1}+b_{2}, \operatorname{ord}_{E_{3}}\left(z_{1}^{b_{1}} z_{2}^{b_{2}}\right)=b_{1}+2 b_{2}$, and $\{2,3\},\{1,3\}$ and $\{1,2\}$ 
are the unique essential multi-indices with respect to $\operatorname{ord}_{E_{1}}, \operatorname{ord}_{E_{2}}$, and $\operatorname{ord}_{E_{3}}$, respectively. Note that this situation cannot happen if $f_{j}$ are all monomials.

Let $q=(1,2,2)$. Then $a\left(f^{q}\right)=\left(z_{1}^{4}-z_{2}^{4}, z_{1}^{4} z_{2}^{2}, z_{1}^{2} z_{2}^{4}\right)$ has four Rees valuations, $\operatorname{ord}_{E_{1}}, \ldots, \operatorname{ord}_{E_{4}}$. To see this, note that after blowing up the origin once, the strict transform of $a\left(f^{q}\right)$ has support at four points $x_{1}, \ldots, x_{4}$. The divisor $E_{j}$ is obtained by further blowing up $x_{j}$ twice. A computation yields that $\{1,2\}$ and $\{1,3\}$ are both $q$-essential with respect to $E_{j}$ for $1 \leq j \leq 4$, whereas $\{2,3\}$ is not $q$-essential. Hence $R(f) \neq R^{q}(f)$.

Note that $\operatorname{det}\left(A_{\mathscr{I}}\right)=0$ is equivalent to that $d z^{a^{i_{1}}} \wedge \cdots \wedge d z^{a^{i_{n}}}$ vanishes identically, which in turn implies that $\mathscr{I}$ is not $p$-essential for any $p \in \mathrm{N}^{m}$. This motivates the following version of Question B.

Question B ${ }^{\prime}$. Is it true that ann $R^{p}(f)$ is independent of $p$ if and only if for any $\mathscr{I}=\left\{i_{1}, \ldots, i_{n}\right\}$, either $f_{\mathscr{I}}$ generates $\mathfrak{a}(f)$ or the form $d f_{i_{1}} \wedge \cdots \wedge d f_{i_{n}}$ vanishes identically.

Let us mention some partial answers to Question $\mathrm{B}^{\prime}$. Theorem $\mathrm{C}$ in [15] asserts that if $\mathfrak{a}(f)$ is a complete intersection ideal, then $R_{\mathscr{A}}(f)$ is a constant times the Coleff-Herrera product $R_{\mathrm{CH}}\left(f_{\mathscr{I}}\right)$ if $f_{\mathscr{I}}$ generates $\mathfrak{a}(f)$ and 0 otherwise. Using this and (1.4) one can check that ann $R^{p}(f)$ is independent of $p$ if $a(f)$ is a complete intersection ideal, generated by say $f_{1}, \ldots, f_{n}$, and moreover for $j>n, f_{j}$ is equal to (a constant times) one of the $f_{k}$ for $1 \leq k \leq n$; compare this to (the discussion right after) Proposition 5.1.

EXAmPLe 5.3. Let $f=\left(z_{1}, z_{2}, z_{1}+z_{2}\right)$. Then $a(f)$ is just the maximal ideal in $\mathscr{O}_{0}^{2}$, which is clearly a complete intersection ideal, and thus by Theorem $\mathrm{C}$ in [15], ann $R(f)=\mathfrak{a}(f)$. Note that any choice of $f_{i}$ and $f_{j}$ generate $a(f)$, so $f$ satisfies the condition in Question $\mathrm{B}^{\prime}$.

Let $p=(3,3,3)$. Observe that $\mathfrak{a}\left(f^{p}\right)=\left(z_{1}^{3}, z_{2}^{3}, z_{1}^{2} z_{2}+z_{1} z_{2}^{2}\right)$ is not a complete intersection ideal. A computation yields that

$$
\begin{aligned}
R_{\{1,3\}}\left(f^{p}\right)=A_{1} \bar{\partial}\left[1 / z_{1}^{5}\right] & \wedge \bar{\partial}\left[1 / z_{2}\right] \\
& +A_{2} \bar{\partial}\left[1 / z_{1}^{4}\right] \wedge \bar{\partial}\left[1 / z_{2}^{2}\right]+A_{3} \bar{\partial}\left[1 / z_{1}^{3}\right] \wedge \bar{\partial}\left[1 / z_{2}^{3}\right],
\end{aligned}
$$

for some constants $A_{1}, A_{2}$, and $A_{3}$. It follows that $R_{\{1,3\}}^{p}(f)=\left(A_{1}+2 A_{2}+\right.$ $\left.A_{3}\right) \bar{\partial}\left[1 / z_{1}\right] \wedge \bar{\partial}\left[1 / z_{2}\right]$. In fact, also the other entries of $R^{p}$ are of this form and so ann $R^{p}=\mathfrak{a}(f)$.

Note that if there is a subsequence $f_{\mathscr{I}}=\left(f_{i_{1}}, \ldots, f_{i_{n}}\right)$ of $f$ such that $V\left(f_{\mathscr{I}}\right)=\{0\}$, then by choosing $p_{j}=1$ if $j \in \mathscr{I}$ and $p_{j} \gg 1$ for $j \notin$ $\mathscr{I}$, the only non-vanishing entry of $R^{p}(f)$ is $R_{\mathscr{I}}^{p}(f)$, which is a constant times $R_{\mathrm{CH}}\left(f_{\mathscr{I}}\right)$. Thus, given that there exists such an $f_{\mathscr{I}}$, ann $R^{p}(f)$ is not 
independent of $p$ as soon as, for example, there is another multi-index $\mathscr{J}$, such that $V\left(f_{\mathscr{J}}\right)=\{0\}$, or as soon as ann $R(f)$ is not a complete intersection ideal. One can, however, not always find such an $f_{\mathscr{I}}$, as the following example shows.

ExAMPLE 5.4. Let $f=\left(f_{1}, f_{2}, f_{3}\right)=\left(z_{1} z_{2}, z_{1}\left(z_{1}+z_{2}\right), z_{2}\left(z_{1}+z_{2}\right)\right)$. Then $V\left(f_{\mathscr{I}}\right)$ is a line through the origin for all $\mathscr{I}=\left\{i_{1}, i_{2}\right\}$; in particular, $V\left(f_{\mathscr{I}}\right) \neq\{0\}$. Moreover, $\mathfrak{a}(f)$ is the (monomial) ideal $\mathfrak{m}^{2}$, where $\mathfrak{m}$ is the maximal ideal in $\mathscr{O}_{0}^{2}$. Thus the only Rees valuation of $a(f)$ is the order of vanishing at the origin and so $R(f)$ can be computed by blowing up the origin once. Note that all multi-indices $\mathscr{I}=\left\{i_{1}, i_{2}\right\}$ are essential. Let $R^{\ell, k}$ denote the current $\bar{\partial}\left[1 / z_{1}^{\ell}\right] \wedge \bar{\partial}\left[1 / z_{2}^{k}\right]$, and let

$$
C_{j}=\frac{1}{2 \pi i} \int \frac{|t|^{2 j} d \bar{t} \wedge d t}{\left(|t|^{2}+|1+t|^{2}+|t(1+t)|^{2}\right)^{2}} .
$$

Then, a computation yields that $R_{\{1,2\}}(f)=-C_{0} R^{3,1}, R_{\{1,3\}}(f)=2 C_{2} R^{1,3}$, and $R_{\{2,3\}}(f)=C_{0} R^{3,1}+2 C_{1} R^{2,2}+C_{2} R^{1,3}$. It follows that ann $R(f)=\mathrm{m}^{3}$.

Let $p=(2,1,1)$. Then $\mathfrak{a}\left(f^{p}\right)$ has two Rees valuations, $\operatorname{ord}_{E_{1}}$ and $\operatorname{ord}_{E_{2}}$, where $E_{1}$ is the exceptional divisor obtained by blowing up the origin once, whereas $E_{2}$ is obtained by further blowing up a point on $E_{1}$ twice. Moreover, $\{2,3\}$ is essential with respect to $E_{1}$ and $\{1,2\}$ and $\{1,3\}$ are essential with respect to $E_{2}$. A computation gives that $R_{\{1,2\}}^{p}(f)=R_{\{1,3\}}^{p}(f)=-1 / 2\left(R^{3,1}-\right.$ $\left.R^{2,2}+R^{1,3}\right)$ and $R_{\{2,3\}}^{p}(f)=A^{3,1} R^{3,1}+A^{2,2} R^{2,2}+A^{1,3} R^{1,3}$, where $A^{i, j}>0$.

Note that $R_{\mathscr{I}}^{p}(f) \neq R_{\mathscr{I}}(f)$, as well as ann $R_{\mathscr{I}}^{p}(f) \neq$ ann $R_{\mathscr{I}}(f)$, for, at least, $\mathscr{I}=\{1,2\},\{1,3\}$. Moreover, note that ann $R(f)$ is strictly included in ann $R^{p}(f)$. Indeed, $\left(A^{2,2}+A^{1,3}\right) z_{1}^{2}+\left(A^{1,3}-A^{3,1}\right) z_{1} z_{2}-\left(A^{3,1}+A^{2,2}\right) z_{2}^{2} \in$ ann $R^{p}(f) \backslash$ ann $R(f)$.

\subsection{Related questions}

Question B could be posed also for the currents (1.5). The following example shows that $e^{p}(f)$ does not necessarily vary with $p$ even if $R^{p}(f)$ and ann $R^{p}(f)$ do.

ExAmple 5.5. Let $z^{A}=\left(z_{1}^{2}, z_{1} z_{2}, z_{2}^{2}\right)$. Then by varying $p$ there are three different possibilities of $p$-essential multi-indices. First, all three multi-indices $\mathscr{I}=\left\{i_{1}, i_{2}\right\}$ could be $p$-essential, which for example is the case for $p=$ $(1,1,1)$. Next, for $p=(1,2,1),\{1,3\}$ is the only $p$-essential multi-index, and for $p=(2,1,1)$, the $p$-essential multi-indices are $\{1,2\}$ and $\{2,3\}$. In the first situation, by [2], $e^{p}\left(z^{A}\right)$ is the Hilbert-Samuel multiplicity of $a\left(z^{A}\right)$, which is equal to $\operatorname{Vol}\left(\mathrm{R}_{+}^{n} \backslash \mathrm{NP}(A)\right)=\left|\operatorname{det}\left(A_{\{1,3\}}\right)\right|=4$. In light of (4.1) it is not hard to check that this is holds true also if $p$ is another weight such that 
all $\mathscr{I}$ are $p$-essential. In the latter two cases, by Section 4.2 , the coefficients $C_{\mathscr{I}}$ are all 1 , when $\mathscr{I}$ is $p$-essential. It follows that $e^{p}=\left|\operatorname{det}\left(A_{\{1,3\}}\right)\right|=4$ and $e^{p}=\left|\operatorname{det}\left(A_{\{1,3\}}\right)\right|+\left|\operatorname{det}\left(A_{\{2,3\}}\right)\right|=2+2$, respectively, so in fact $e^{p}\left(z_{A}\right)$ is independent of $p$.

One can also ask in what sense $R_{\mathscr{I}}^{p}(f)$ and ann $R_{\mathscr{I}}^{p}(f)$ depend on $p$, once $\mathscr{I}$ is $p$-essential. In the monomial case ann $R_{\mathscr{I}}^{p}(f)$ is fix as long as $\mathscr{I}$ is essential but the coefficient $C_{\mathscr{I}}$ in (1.6) vary in general. Indeed, in Example 5.5 above, in the first case, for $p=(1,1,1), C_{\mathscr{I}}$ are all strictly between 0 and 1 , whereas in the latter cases they are either 0 or 1 . In general, also ann $R_{\mathscr{I}}^{p}(f)$ varies with $p$, see Example 5.4 above. Computations, such as in Example 5.4, suggest that in general there may be infinitely many different annihilator ideals ann $R_{\mathscr{I}}^{p}(f)$ and ann $R^{p}(f)$ as $p$ varies over $\mathbf{N}^{m}$. This contrasts the monomial case, where there are always finitely many different ideals ann $R^{p}(f)$.

\section{REFERENCES}

1. Andersson, M., Residue currents and ideals of holomorphic functions, Bull. Sci. Math. 128 (2004), 481-512.

2. Andersson, M., Residues of holomorphic sections and Lelong currents, Ark. Mat. 43 (2005), 201-219.

3. Andersson, M., and Götmark, E., Explicit representation of membership in polynomial ideals, Math. Ann. 349 (2011), 345-365.

4. Andersson, M., Samuelsson, H., and Sznajdman, J., On the Briançon-Skoda theorem on a singular variety, Ann. Inst. Fourier 60 (2010), 417-432.

5. Berenstein, C., Vidras, A., and Yger, A., Analytic residues along algebraic cycles, J. Complexity 21 (2005), no. 1, 5-42.

6. Berenstein, C., and Yger, A., Analytic residue theory in the non-complete intersection case, J. Reine Angew. Math. 527 (2000), 203-235.

7. Berenstein, C. A., Gay, R., Vidras, A., and Yger, A., Residue Currents and Bezout Identities, Progress in Math. 114, Birkhäuser, Basel 1993.

8. Briançon, J., and Skoda, H., Sur la clôture intégrale d'un idéal de germes de fonctions holomorphes en un point de $\mathbf{C}^{n}$, C. R. Acad. Sci. Paris (A) 278 (1974), 949-951.

9. Coleff, N., and Herrera, M., Les courants résiduels associcés à une forme méromorphe, Lecture Notes in Math. 633, Springer, Berlin 1978.

10. Dickenstein, A., and Sessa, C., Canonical representatives in moderate cohomology, Invent. Math. 80 (1985), 417-434.

11. Fulton, W., Introduction to Toric Varieties, Annals of Math. Studies 131, Princeton Univ. Press, Princeton, NJ 1993.

12. Griffiths, P., and Harris, J., Principles of Algebraic Geometry, Pure Appl. Math., WileyInterscience, New York 1978.

13. Hartshorne, R., Algebraic Geometry, Graduate Texts in Math. 52, Springer, New York 1977.

14. Huneke, C., and Swanson, I., Integral Closure of Ideals, Rings, and Modules, London Math. Soc. Lecture Note 336, Cambridge Univ. Press, Cambridge 2006.

15. Jonsson, M., and Wulcan, E., On Bochner-Martinelli residue currents and their annihilator ideals, Ann. Inst. Fourier 59 (2009), 2119-2142. 
16. Lazarsfeld, R., Positivity in Algebraic Geometry I \& II, Ergebn. Math. Grenzgeb. (3) 48 \& 49, Springer, Berlin 2004.

17. Lipman, J., Residues and Traces of Differential Forms via Hochschild Homology, Contemporary Math. 61, Amer. Math. Soc., Providence, RI 1987.

18. Passare, M., Residues, currents, and their relation to ideals of holomorphic functions, Math. Scand. 62 (1988), 75-152.

19. Passare, M., Tsikh, A., and Yger, A., Residue currents of the Bochner-Martinelli type, Publ. Mat. 44 (2000), 85-117.

20. Teissier, B., Variétés polaires II, Multiplicités polaires, sections planes, et conditions de Whitney, pp. 314-491 in: Algebraic Geometry, Proc. La Rábida 1981, Lecture Notes in Math. 961, Springer, Berlin 1982.

21. Teissier, B., Monômes, volumes et multiplicités, pp. 127-141 in: Introduction à la théorie des singularités II, Travaux en Cours 37, Hermann, Paris 1988.

22. Vidras, A., and Yger, A., On some generalizations of Jacobi's residue formula, Ann. Sci. École Norm. Sup. (4) 34 (2001), 131-157.

23. Wulcan, E., Residue currents of monomial ideals, Indiana Univ. Math. J. 56 (2007), 365-388.

24. Yger, A., Aspects opérationnels de la théorie des résidus hors du cadre intersection complète, pp. 182-204 in: Géométrie complexe II, Aspects contemporains dans les mathématiques et la physique, Hermann, Paris 2004.

DEPARTMENT OF MATHEMATICS

CHALMERS UNIVERSITY OF TECHNOLOGY AND THE UNIVERSITY OF GOTHENBURG

SE-412 96 GÖTEBORG

SWEDEN

E-mail: wulcan@chalmers.se 